\title{
A game-theoretical model for a controlled process of heat transfer
}

\author{
O.A. Malafeyev
}

Saint Petersburg University, Department of Applied

Mathematics-Processes of Control

Frunze street 6, flat 225, St. Petersburg 196070, Russia.

\section{M.S. Troeva}

Yakut Research Institute for Applied Mathematics and Informatic

Petra Alekseeva 8/1, flat 16, Yakutsk 677000, Russia.

\begin{abstract}
The problem of finding an optimal technological mode for a controlled process of heat transfer is considered in this paper. This problem is formulated as a differential twoperson zero-sum game of a technologist against 'nature'.

To solve the problem considered the numerical method based on the dynamic programming method and the finite difference method is proposed.
\end{abstract}

\section{Keywords}

Differential game, numerical method, piecewise-programmed strategies, dynamic programming, finite difference method.

The problem of finding an optimal technological mode for a controlled process of heat transfer is considered in this paper.

A rectangular solid body in a medium with variable temperature is considered. The temperature field is controlled by heat (energy) supply in some way. The mathematical description of this action is described by a parameter $w$, controlled by the technologist. This parameter is an element of a set $W$ of all control parameters. The temperature field of the body depends also on the state $v$ of the medium. This is a "nature's" control parameter which belongs to a set $V$ of all control parameters.

The quality of the process's control is estimated by a payoff function $H$.

The problem of choosing control function $w=w(t), t \in[0, T], w(t) \in W$, which guarantees the optimal value of the quality index $H$ under any possible "nature's" function $v$ arises. It is interesting for example to change the temperature field from initial state $u_{0}$ to given final state $u_{T}$ with minimal energy expenses under uncertain "nature's" conditions by choosing control function $w(\cdot)$.

So, the problem can be formulated as a differential two-person zero-sum game $\Gamma\left(u_{0}, T\right)$ 
with initial position $u_{0}$ and duration $T$ in the space $C_{\bar{\Omega}}$ of continuous functions with the domain $\bar{\Omega}=\left[0, l_{1}\right] \times\left[0, l_{2}\right]$.

The dynamics of the game $\Gamma\left(u_{0}, T\right)$ is described by the following boundary value problem for the heat transfer equation:

$$
\begin{aligned}
c \frac{\partial u}{\partial t} & =\frac{\partial}{\partial x_{1}}\left(\lambda \frac{\partial u}{\partial x_{1}}\right)+\frac{\partial}{\partial x_{2}}\left(\lambda \frac{\partial u}{\partial x_{2}}\right), x_{1} \in\left(0, l_{1}\right), x_{2} \in\left(0, l_{2}\right), \\
\lambda \frac{\partial u}{\partial x_{1}} & =\alpha_{1}(u-v), x_{1}=0, x_{2} \in\left[0, l_{2}\right], t>0 \\
-\lambda \frac{\partial u}{\partial x_{1}} & =\alpha_{1}(u-v), x_{1}=l_{1}, x_{2} \in\left[0, l_{2}\right], t>0, \\
\lambda \frac{\partial u}{\partial x_{2}} & =\alpha_{1}(u-v), x_{1} \in\left[0, l_{1}\right], x_{2}=0, t>0 \\
-\lambda \frac{\partial u}{\partial x_{2}} & =\alpha_{2}(u-w), x_{1} \in\left[0, l_{1}\right], x_{2}=l_{2}, t>0 \\
u\left(x_{1}, x_{2}, 0\right) & =u_{0}\left(x_{1}, x_{2}\right), x_{1} \in\left[0, l_{1}\right], x_{2} \in\left[0, l_{2}\right], t=0
\end{aligned}
$$

where $u(x, t)$ - the temperature at the point $x=\left(x_{1}, x_{2}\right) \in \bar{\Omega}$ at the moment $\mathrm{t}, c=$ $c(x, t)$ - volume heat capacity, $\lambda=\lambda(x, t)$ - heat conductivity coefficient, $\alpha_{1}, \alpha_{2}$ - heat exchange coefficients; $w, v$ - the control's parameters of the players, $P$ (technologist) and $E$ (nature), respectively; $w \in W \subset R^{p}, v \in V \subset R^{q} ; W$ and $V$ - are compact sets in Euclidean spaces $R^{p}$ and $R^{q}$ respectively.

The continuous function $w=w(t)(v=v(t))$, satisfying the condition $w(t) \in W(v(t) \in$ $V$ ) for all $t \in[0, T]$ is called the admissible control of the player $P(E)$.

It is known (Tichonov and Samarsky, 1977), that for any finite $T<\infty$ for any admissible controls $w(t), t \in[0, T]$ and $v(t), t \in[0, T]$ there exists the unique solution $u(t)=$ $u\left(u_{0}, t, w(t), v(t)\right)$ of the problem (1)-(6) under any $u_{0} \in C_{\bar{\Omega}}$ and $t \in[0, T]$.

At any moment $t$ of the game the players are informed of the state of the game $u(t)$, initial moment $t_{0}=0$, terminal time $T$ and the dynamics of the game.

For definiteness we will consider the above formulated problem. At the moment $T$ the player $E$ gets the payoff $H(\cdot)$ from the player $P$ :

$H(u(\cdot))=\int_{0}^{T} h(u(t), w(t), v(t), t) d t$,

where $u(\cdot)$ is a trajectory of the process, corresponding to admissible controls $w(\cdot)$ and $v(\cdot)$ on the interval $[0, T], h(u, w, v, t)$ is a continuous function bounded on the bounded sets. This function characterizes the energy expenses.

The aim of the player $P$ is to minimize $H(\cdot)$ and the aim of the player $E$ is contrary.

Let us cite (Malafeyev, 1993) the definition of strategies of players $P$ and $E$ in the game $\Gamma\left(u_{0}, T\right)$.

Definition $1 A$ strategy $\varphi(\psi)$ of player $P(E)$ in the game $\Gamma\left(u_{0}, T\right)$ is the pair $\left(\sigma_{1}, K_{\sigma_{1}}\right)$ $\left(\left(\sigma_{2}, K_{\sigma_{2}}\right)\right)$, where $\sigma_{1}\left(\sigma_{2}\right)$ is an arbitrary finite partition of the interval $[0, T]$, while $K_{\sigma_{1}}$ $\left(K_{\sigma_{2}}\right)$ is a mapping which associates an admissible control $w_{i}(\tau), \tau \in\left[t_{i}, t_{i+1}\right)\left(v_{j}(\tau), \tau \in\right.$ 
$\left.\left[t_{j}, t_{j+1}\right)\right)$ with the information state of player $P(E)$ in the moment $t_{i} \in \sigma_{1}, i=0, \ldots, N_{\sigma_{1}}-1$ $\left(t_{j} \in \sigma_{2}, j=0, \ldots, N_{\sigma_{2}}-1\right)$.

The set of strategies of the player $P(E)$ in the game $\Gamma\left(u_{0}, T\right)$ is denoted by $\Phi(\Psi)$.

The trajectory of the game $\chi(\varphi, \psi)$ is uniquely defined by the standard way for every strategy pair $(\varphi, \psi) \in \Phi \times \Psi$ under initial position $u_{0}$.

The payoff function of the player $E$ for every strategy pair $(\varphi, \psi)$ is defined as follows:

$$
K\left(u_{0}, \varphi, \psi\right)=H(\chi(\varphi, \psi)(\cdot))
$$

where $\chi(\varphi, \psi)(\cdot)$ is a trajectory of the game $\Gamma\left(u_{0}, T\right)$, corresponding to the $(\varphi, \psi)$.

Making use of the results of the dynamic games theory in complete metric spaces (Malafeyev, 1993), the existence of $\varepsilon$-equilibrium points in auxiliary approximate games of perfect information with discrimination of a player (upper and lower games $\bar{\Gamma}^{\sigma}\left(u_{0}, T\right)$ and $\underline{\Gamma}^{\sigma}\left(u_{0}, T\right)$ with discrimination of players $P$ and $E$, respectively) is proved for the class of the piecewise-programmed strategies.

To find the optimal mode for the controlled process of heat transfer the numerical method based on the dynamic programming method (Bellman, 1960) and the finite difference method (Samarsky, 1989) is proposed.

On the domain $\bar{\Omega}=\left[0, l_{1}\right] \times\left[0, l_{2}\right]$ we construct the uniform net with steps $h_{1}$ on $x_{1}$ and $h_{2}$ on $x_{2}$

$$
\begin{aligned}
\bar{\omega}_{h}= & \left\{x_{1}^{(i)}=i h_{1}, i=0, \ldots, N_{1} ; x_{1}^{(0)}=0, x_{1}^{\left(N_{1}\right)}=l_{1} ;\right. \\
& \left.x_{2}^{(k)}=k h_{2} ; k=0, \ldots, N_{2} ; x_{2}^{(0)}=0, x_{2}^{\left(N_{2}\right)}=l_{2}\right\},
\end{aligned}
$$

where $h=\left(h_{1}, h_{2}\right)$.

On the interval $\left[t_{s}, t_{s+1}\right], s=\overline{0, N_{\sigma}-1}$ we construct the uniform net with step $\delta$

$\bar{\omega}_{\delta}=\left\{\tau_{j}=j \delta, j=\overline{0, N_{3}} ; \tau_{0}=t_{s}, \tau_{N_{3}}=t_{s+1}\right\}$.

Here $t_{s} \in \sigma$, where $\sigma$ is the time interval partition

$\sigma=\left\{t_{0}=0, t_{1}, \ldots, t_{N_{\sigma}}=T\right\}$.

Let us denote by $y_{i k}^{j}$ the function defined on the net $\bar{\omega}_{h \delta}=\bar{\omega}_{h} \times \bar{\omega}_{\delta}$.

For approximate description of the attainability set at the moment $t_{s+1}$ under any pair of admissible controls $w_{s}(t), v_{s}(t), t \in\left[t_{s}, t_{s+1}\right)$ for the problem (1)-(6) we construct purely implicit locally one-dimensional difference scheme (Samarsky, 1989):

$$
\begin{aligned}
& c_{0, k}^{j+1 / 2} \frac{y_{0, k}^{j+1 / 2}-y_{0, k}^{j}}{\delta}= \\
& =2 \lambda_{1 / 2, k}^{j+1 / 2} \frac{y_{1, k}^{j+1 / 2}-y_{0, k}^{j+1 / 2}}{h_{1}^{2}}-2 \alpha_{1} \frac{y_{0, k}^{j+1 / 2}-v_{s}}{h_{1}}, \quad i=0, \\
& c_{i, k}^{j+1 / 2} \frac{y_{i, k}^{j+1 / 2}-y_{i, k}^{j}}{\delta}=
\end{aligned}
$$




$$
\begin{aligned}
& =\lambda_{i+1 / 2, k}^{j+1 / 2} \frac{y_{i+1, k}^{j+1 / 2}-y_{i, k}^{j+1 / 2}}{h_{1}^{2}}-\lambda_{i-1 / 2, k}^{j+1 / 2} \frac{y_{i, k}^{j+1 / 2}-y_{i-1, k}^{j+1 / 2}}{h_{1}^{2}}, i=\overline{1, N_{1}-1}, \\
& c_{N_{1}, k}^{j+1 / 2} \frac{y_{N_{1}, k}^{j+1 / 2}-y_{N_{1}, k}^{j}}{\delta}= \\
& =-2 \alpha_{1} \frac{y_{N_{1}, k}^{j+1 / 2}-v^{s}}{h_{1}}-2 \lambda_{N_{1}-1 / 2, k}^{j+1 / 2} \frac{y_{N_{1}, k}^{j+1 / 2}-y_{N_{1}-1, k}^{j+1 / 2}}{h_{1}^{2}}, i=N_{1}, \\
& k=\overline{0, N_{2}} \\
& j=\overline{0, N_{3}-1} \text {, } \\
& c_{i, 0}^{j+1} \frac{y_{i, 0}^{j+1}-y_{i, 0}^{j+1 / 2}}{\delta}= \\
& =2 \lambda_{i, 1 / 2}^{j+1} \frac{y_{i, 1}^{j+1}-y_{i, 0}^{j+1}}{h_{2}^{2}}-2 \alpha_{1} \frac{y_{i, 0}^{j+1}-v^{s}}{h_{2}}, k=0, \\
& c_{i, k}^{j+1} \frac{y_{i, k}^{j+1}-y_{i, k}^{j+1 / 2}}{\delta}= \\
& =\lambda_{i, k+1 / 2}^{j+1} \frac{y_{i, k+1}^{j+1}-y_{i, k}^{j+1}}{h_{2}^{2}}-\lambda_{i, k-1 / 2}^{j+1} \frac{y_{i, k}^{j+1}-y_{i, k-1}^{j+1}}{h_{2}^{2}}, k=\overline{1, N_{2}-1}, \\
& c_{i, N_{2}}^{j+1} \frac{y_{i, N_{2}}^{j+1}-y_{i, N_{2}}^{j+1 / 2}}{\delta}= \\
& =-2 \alpha_{2} \frac{y_{i, N_{2}}^{j+1}-w_{s}}{h_{2}}-2 \lambda_{i, N_{2}-1 / 2}^{j+1} \frac{y_{i, N_{2}}^{j+1}-y_{i, N_{2}-1}^{j+1}}{h_{2}^{2}}, k=N_{2}, \\
& i=\overline{0, N_{1}} \text {, } \\
& j=\overline{0, N_{3}-1} \text {, }
\end{aligned}
$$

where

$y_{i, k}^{N_{3}, 0}=u_{0}\left(x_{1}^{(i)}, x_{2}^{(k)}\right), i=\overline{0, N_{1}}, k=\overline{0, N_{2}}, j=0, s=0$.

The constructed difference scheme (9)-(15) is solved by the sweep method (Samarsky, 1989).

The operator form of the difference scheme (9)-(15) is following:

$$
\begin{aligned}
& \mathcal{D}_{\alpha}^{j+1 / 2} y_{\delta \alpha}+A_{\alpha}^{j+\alpha / 2} y^{j+\alpha / 2}=\varphi_{\alpha}^{j+\alpha / 2}, \alpha=1,2 ; j=\overline{0, N_{3}-1} \\
& y_{i, k}^{0}=y_{i, k}^{N_{3}, s}, i=\overline{0, N_{1}}, k=\overline{0, N_{2}}, j=0, s=\overline{0, N_{\sigma}-1}
\end{aligned}
$$

where

$y_{\delta \alpha}=\frac{y^{j+\alpha / 2}-y^{j+(\alpha-1) / 2}}{\delta}$

$y_{i, k}^{N_{3}, 0}=u_{0}\left(x_{1}^{(i)}, x_{2}^{(k)}\right), i=\overline{0, N_{1}}, k=\overline{0, N_{2}}, j=0, s=0$. 
The following statements about the stability and the convergence of the difference scheme (9)-(15) are valid.

Theorem 1 Let us consider the operators $\mathcal{D}_{\alpha}^{j+\alpha / 2} \geq c_{0} E$ and $A_{\alpha}^{j+\alpha / 2}=A_{\alpha}^{j+\alpha / 2 *} \geq c_{1} \Lambda$ that are positive defined and the operator $A_{\alpha}^{j+\alpha / 2}$ that satisfies the Lipschitz condition

$A_{\alpha}^{j+\alpha / 2} \leq\left(1+c_{3} \delta\right) A_{\alpha}^{j}, \alpha=1,2 ; j=\overline{0, N_{\sigma}-1}$.

Then locally one-dimensional difference scheme (9)-(15) is absolutely stable and the a priori estimate is valid:

$\left|\left[y^{n+1}\right]\right|_{A_{2}^{n+1}} \leq M_{1}\left\{\left|\left[y^{0}\right]\right|_{A_{1}^{0}}+\sum_{j=0}^{n} \sqrt{\frac{\delta}{4 \varepsilon}} \sum_{\alpha=1}^{2}\left|\left[\varphi_{\alpha}^{j+\alpha / 2}\right]\right|\right\}$,

where $M_{1}=e^{0.5 c_{3} t_{0}}$.

Theorem 2 For $|h| \rightarrow 0, \delta \rightarrow 0$ the solution of the difference scheme (9)-(15) converges to the solution of the problem (1)-(6) by the rate of order $o\left(\delta+|h|^{2}\right)$ and the following estimate is valid:

$\left|\left[z^{n+1}\right]\right|_{A_{2}^{n+1}} \leq M_{2}\left\{\sum_{j=0}^{n} \sqrt{\frac{\delta}{4 \varepsilon}} \sum_{\alpha=1}^{2}\left|\left[\psi_{\alpha}^{j+\alpha / 2}\right]\right|\right\}$,

where $|h|^{2}=h_{1}^{2}+h_{2}^{2}, M_{2}=$ const.

Example. Let us consider the differential two-person zero-sum game $\Gamma\left(u_{0}, T\right)$ which describes the process of heating of the bar in a medium with variable temperature.

The temperature $w$ on the left side of the bar is controlled by the technologist (the player $P$ ). The control parameter $w$ is an element of a set $\left[W_{1}, W_{2}\right]$ of all control parameters. The heat exchange between the bar and the medium of temperature $v$ (where $v$ is a "nature's" (the player $E$ ) control parameter) takes place on the right side of the bar. The control parameter $v$ is an element of a set $\left[V_{1}, V_{2}\right]$ of all control parameters.

The dynamics of the game $\Gamma\left(u_{0}, T\right)$ is described by the following boundary value problem for the heat transfer equation:

$$
\begin{aligned}
c \frac{\partial u}{\partial t} & =\frac{\partial}{\partial x}\left(\lambda \frac{\partial u}{\partial x}\right), x \in(0, l), t>0 \\
u(0, t) & =w, x=0, t>0 \\
-\lambda \frac{\partial u}{\partial x} & =\alpha(u-v), x=l, t>0 \\
u(x, 0) & =u_{0}(x), x \in[0, l], t=0
\end{aligned}
$$

The problem is to support given temperature $u_{\xi}(t), t \in[0, T]$ at the fixed bar point $\xi \in(0, l)$ under any uncertain "nature's" conditions by means of choosing a control function $w=w(\cdot)$. 
Thus, the player $E$ gets the following payoff from the player $P$ at the terminal moment $T$ of the game:

$H=\sqrt{\frac{1}{T} \int_{0}^{T}\left(u(\xi, t)-u_{\xi}(t)\right)^{2} d t}$,

where $u(\xi, t)$ is a temperature at the bar point $\xi \in(0, l)$ at the moment $t \in[0, T]$.

The aim of the player $P$ is to minimize $H$ and the aim of the player $E$ is contrary.

The method suggested above is used for numerical solving for the described problem.

We assume the bar being uniform. Then one may consider the coefficients $c, \lambda$ as constants. The numerical experiments were realized for the following input data: $W_{1}=10^{\circ} \mathrm{C}$, $W_{2}=20^{\circ} \mathrm{C} ; V_{1}=1^{\circ} \mathrm{C}, V_{2}=20^{\circ} \mathrm{C}$; the length of bar $l=1 \mathrm{~m}, \xi=l / 2$; heat conductivity coefficient $\lambda=45.400 \mathrm{~W} /\left(\mathrm{m}^{\circ}{ }^{\circ} \mathrm{C}\right)$, density $\rho=7900 \mathrm{~kg} / \mathrm{m}^{3}$, specific heat capacity $c_{p}=462$ $\mathrm{J} /\left(\mathrm{kg} \cdot{ }^{0} \mathrm{C}\right)$; heat exchange coefficient $\alpha=50 \mathrm{~W} /\left(\mathrm{m}^{2}{ }^{\circ} \mathrm{C}\right)$; the initial temperature distribution $u_{0}(x)=12^{\circ} \mathrm{C}$; the time of heating $T=10$ hour.

The following function $u_{\xi}(t)$ has been considered at the fixed bar point $\xi$ :

$u_{\xi}(t)=13+\sin \left(2 \pi t / T-\frac{\pi}{2}\right), \quad t \in[0, T]$.

The value functions of the upper and lower games are:

$\operatorname{Val}\left(\bar{\Gamma}^{\sigma}\left(u_{0}, T\right)\right)=0.149$

$\operatorname{Val}\left(\underline{\Gamma}^{\sigma}\left(u_{0}, T\right)\right)=0.111$.

The results of numerical experiments are given on the Figures 1-3.

\section{REFERENCES}

Tichonov, A.N., Samarsky, A.A. (1977) Equations of Mathematical Physics. Nauka, Moscow (Russian).

Malafeyev, O.A. (1993) Dynamical control systems of conflict. St. Petersburg (Russian). Bellman, R. (1960) Dynamic programming. IL, Moscow (Russian).

Samarsky, A.A. (1989) Difference schemes theory. Nauka, Moscow (Russian). 


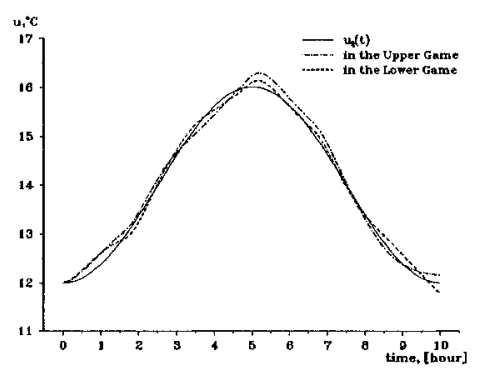

Figure 1 The Temperature at the Bar Point $\xi$.

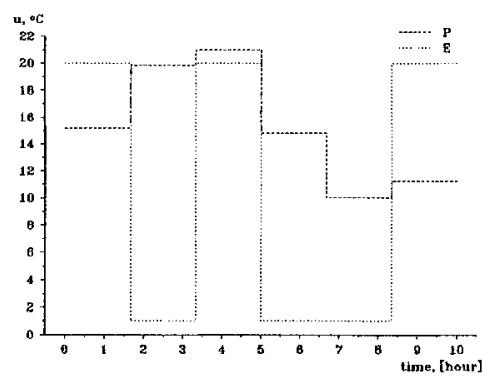

Figure 2 The Upper Game: The Optimal Control of the Players. 


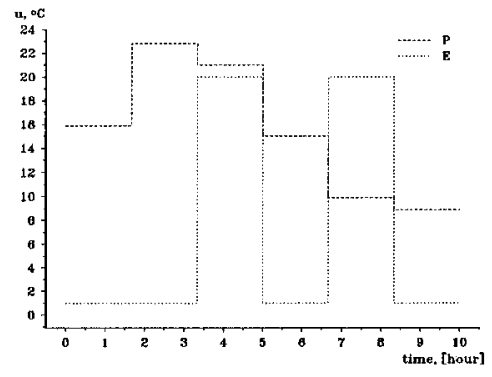

Figure 3 The Lower Game: The Optimal Control of the Players. 